\title{
NEW APPROACH TO INVESTIGATION OF NUCLEI
}

\author{
E. G. Drukarev ${ }^{1}$, a , M. G. Ryskin ${ }^{1}$, and V. A. Sadovnikova ${ }^{1}$ \\ ${ }^{1}$ Petersburg Nuclear Physics Institute, National Research Center "Kurchatov Institute", St. Petersburg \\ 188300, Russia
}

\begin{abstract}
We demonstrate that the sum rules of quantum chromodynamics provide a consistent formalism for solving various problems of nuclear physics.
\end{abstract}

\section{Introduction}

It is well known that the standard approach to the description of nucleons in nuclear matter, based on the concept of interactions between point-like nucleons, faces many problems connected with short distances. On the other hand the strong interaction is known to become increasingly simple at short distances, due to the asymptotic freedom of Quantum Chromodynamics (QCD). It is tempting to use this feature of QCD in building nuclear forces. One should take into account, however, that due to the spontaneous breakdown of the of the chiral symmetry of QCD, the expectation values of some QCD operators (condensates) obtain nonzero values. Our approach is to extend the method of QCD sum rules (SR) to systems with a finite density of the baryon quantum number. The SR approach was suggested and was successfully applied to the calculation of the static characteristics of mesons and nucleons in vacuum [1].

\section{Milestones of the approach}

The approach is based on the dispersion relations for the function $\Pi_{0}\left(q^{2}\right)$, describing the space-time propagation of a system with four-momentum $q$ and which carries the quantum numbers of the hadron. At $q^{2} \rightarrow-\infty$ the function $\Pi_{0}\left(q^{2}\right)$ just describes a system of three free quarks (i.e. asymptotic freedom) moving, however, in the QCD vacuum. At $q^{2}>0, \Pi_{0}\left(q^{2}\right)$ obtains an imaginary part describing the observable hadrons. The equations which follow from the dispersion relations require further evaluation (via the Borel transform). The approach is focused on studies of the lowest state. Due to the asymptotic freedom of QCD it was possible to connect the strong interactions at distances of the order of the confinement radius with those at much smaller distances, where the interactions are determined by QCD condensates. The left-hand side (LHS) of the SR was presented as a power series in $1 / q^{2}$, with the QCD condensates as the coefficients of the expansion. The lowest order terms thus contain condensates of the lowest dimension $d$. The most important condensates are the vacuum expectation values of the quark operators $\bar{q} q(d=3)$ and $\bar{q} q \bar{q} q(d=6)$ and of the gluon operators $G_{\mu \nu} G^{\mu \nu}(d=4)$. On the right hand side (RHS) of the SR one writes down the contribution of the

\footnotetext{
a e-mail: drukarev@thd.pnpi.spb.ru
} 
lowest pole explicitly, while the higher states are usually approximated by a continuum. All static and some of dynamical characteristics of the hadrons were presented in terms of QCD condensates. Thus we expect that the parameters of the nucleon in nuclear matter can be expressed in terms of the in-medium values of the QCD condensates.

The generalization of the SR method to the case of a nucleon in nuclear matter, which was initiated in [2], is not straightforward. Considering the nuclear matter as a system of $A \rightarrow \infty$ nucleons with momenta $p_{i}(i \leq A)$ we introduce momentum $P=\sum_{i} p_{i} / A$ with $P=(m, 0)$ in the rest frame of the matter (where $m \approx m_{N}, m_{N}$ is the nucleon mass). The spectrum of the function $\Pi_{m}(q, P)$ is much more complicated than that of the vacuum function $\Pi_{0}\left(q^{2}\right)$. One of the main problems was the choice of variables to enable a separation of the singularities connected with the in-medium nucleon from those connected with the medium itself. Excitations of the matter manifest themselves as singularities in the variable $s=(P+q)^{2}$. Thus the separation can be done by considering $\Pi_{m}\left(q^{2}, s\right)$ at $s=\operatorname{const}\left(\approx 4 m_{N}^{2}\right)$. The SR in matter are based on dispersion relations for the functions $\Pi_{m}^{i}\left(q^{2}, s\right)$ at fixed $s$. The three functions $(i=1,2,3)$ correspond to the three structures of the function $\Pi_{m}$ which are proportional to $\gamma_{\mu} q^{\mu}, \gamma_{\mu} P^{\mu}$ and to the unit matrix.

As in the vacuum case the LHS of the SR is a power series in $1 / q^{2}$, but the coefficients are now the in-medium expectation values of QCD operators. Some of the operators are the same as in the vacuum case, obtaining, however new expectation values since now they are averaged over the ground state of the matter. These are, for example, the quark operators $\bar{q} q$ and the gluon operators $G_{\mu \nu}^{a} G^{a \mu \nu}$. There are also the operators which only obtain nonzero values in the nuclear medium. The most important one is the vector operator $\bar{q} \gamma_{0} q$.

First, we may try to calculate the density dependence of the nucleon vector self-energy $\Sigma_{V}$ and of the Dirac effective mass $m_{N}^{*}$ (or the scalar self-energy $\Sigma_{S}=m_{N}^{*}-m$ ) in symmetric nuclear matter. The lowest dimension $(d=3)$ condensates are the vector and scalar condensates

$$
v(\rho)=\left\langle M\left|\sum_{i} \bar{q}^{i} \gamma_{0} q^{i}\right| M\right\rangle ; \quad \kappa(\rho)=\left\langle M\left|\sum_{i} \bar{q}^{i} q^{i}\right| M\right\rangle,
$$

where $|M\rangle$ is the ground state of the matter. Here $\rho$ is the density of the matter, and $i=1,2$ correspond to the $u$ and $d$ quark flavors. Due to the conservation of the vector current, the vector condensate is exactly proportional to the density $\rho$ via

$$
v(\rho)=v_{N} \rho ; \quad v_{N}=\left\langle N\left|\sum_{i} \bar{q}^{i} \gamma_{0} q^{i}\right| N\right\rangle .
$$

Here $|N\rangle$ is the free nucleon state, and $v_{N}=3$ is just the number of valence quarks in the nucleon. The scalar condensate can be written as

$$
\kappa(\rho)=\kappa(0)+\kappa_{N} \rho+S(\rho) .
$$

Here $\kappa(0)$ is the vacuum value of the scalar condensate, which is the most important parameter in the vacuum SR, and $\kappa_{N}=\left\langle N\left|\sum_{i} \bar{q}^{i} q^{i}\right| N\right\rangle$, with $|N\rangle$ describing the free nucleon. The term $S(\rho)$ is due to the interaction of the nucleons of the matter. It contains higher powers of the Fermi momentum $p_{F} \sim \rho^{1 / 3}$.

\section{QCD sum rules in gas approximation}

Putting $S=0$ we obtain $\kappa(\rho)$ in the gas approximation. A separate analysis carried out by several groups has demonstrated that $S(\rho)$ is small for densities close to the saturation point. Thus it is 
reasonable to start with the SR in the gas approximation. The expectation value $\kappa_{N}$ can be expressed in terms of the pion nucleon $\sigma$ term

$$
\kappa_{N}=\frac{2 \sigma}{m_{u}+m_{d}},
$$

(where $m_{u, d}$ are the quark current masses), while $\sigma$ can be expressed in terms of the pion-nucleon elastic scattering amplitude. Also, the gluon condensate $(d=4) g(\rho)=\left\langle M\left|\alpha_{s} / \pi G_{a \mu \nu} G^{a \mu \nu}\right| M\right\rangle$ in the gas approximation can be written as $g(\rho)=g(0)+g_{N} \rho$, where $g_{N} \approx-8 / 9 m_{N}$ can be obtained by averaging the trace of the QCD energy-momentum tensor over the nucleon state. Thus the LHS of the SR can be either calculated in a model-independent way or related to observables.

The SR give $\Sigma_{V}=240 \mathrm{MeV}$ and $m_{N}^{*}-m_{N}=-370 \mathrm{MeV}$ for $\sigma=60 \mathrm{MeV}$. Due to the uncertainty in the experimental value of $\sigma$, these values are not very accurate. Moreover, the nucleon in matter moves in a superposition of high-energy (several hundreds of $\mathrm{MeV}$ ) fields with vector and scalar terms providing positive and negative contributions to the potential energy. This is consistent with the relativistic models currently employed in nuclear physics. Turning to the meson-exchange picture of the nucleon interaction we can say that in the SR approach the exchange by strongly correlated quark systems (mesons) is expressed in terms of weakly correlated quark systems (including radiative corrections) with the same quantum numbers.

The four-quark condensates $(d=6)$ are the most important among those of higher dimensions. For several years a lack of information about their values has been an obstacle to the development of this approach. The calculation required model assumptions about the quark structure of nucleon. It was later demonstrated that inclusion of the four-quark condensates does not alter qualitatively the results obtained by inclusion of terms of lowest dimension only.

This approach was used for a description of nucleons in asymmetric nuclear matter. It was successfully employed also for calculation of the nucleon axial coupling constant in nuclear matter. The SR approach proved itself to be a reasonable tool for solving problems which, in the framework of traditional nuclear physics, appeared to be complicated. For example, the problem of neutron-proton mass splitting in isotope symmetric nuclear matter required the introduction of charge-symmetry violating nuclear forces. In the SR framework it requires inclusion of nonzero values of the current quark masses $m_{u, d}$ and of the charge-symmetry breaking condensate $\langle M|\bar{u} u-\bar{d} d| M\rangle$. The SR approach provided at least a qualitative explanation of the Nolen-Schiffer anomaly.

The method enables an investigation of the internal structure of the nucleon. Such problems are unaccessible in traditional nuclear physics. The SR approach was applied to the calculation of the difference between the deep inelastic structure functions of the nucleon in nuclear matter and those of the free one, providing a description of some features of the EMC effect.

\section{Beyond the gas approximation}

Going beyond the gas approximation we must include the nonlinear term $S(\rho)$ in the expression for the scalar condensate. It is produced by the meson cloud, created by the interacting nucleons of the matter. Under certain reasonable assumptions the matrix element $n_{q}=\langle h|\bar{q} q| h\rangle$ counts the total number of quarks and antiquarks in a hadron $h$. For the pion this matrix element can be expressed in terms of the vacuum expectation value by means of the current algebra. It was found that $n_{q} \approx 12$ in this case, while $n_{q} \approx 2$ due to other mesons. Thus we expect the pion cloud to provide the leading contribution to $S(\rho)$. A simplified calculation which does not include the pion renormalization in the nuclear medium leads to the following expression for the binding energy per nucleon

$$
\varepsilon(z)=23 z^{2 / 3}+\left(215-21 \kappa_{N}\right) z+54 z^{4 / 3}[\mathrm{MeV}] .
$$


Here $z=\rho / \rho_{0}$ with $\rho_{0}=0.16 \mathrm{Fm}^{-3}$ is the phenomenological value of the nuclear density. One finds that $\varepsilon^{\prime}(z)=0$ at $z=1$ if $\sigma=64 \mathrm{MeV}$, which is consistent with experimental data. At this point $\Sigma_{V}=260 \mathrm{MeV}, \Sigma_{S}=-310 \mathrm{MeV}$, however one should not take this too seriously. In obtaining the nucleon self-energies, the calculations of the potential energy require somewhat higher accuracy since it is a result of the subtraction of two large values. Thus a reasonable value of the potential energy is a surprise. Also, the result is very sensitive to the value of the $\sigma$ term.

However, this result may be a sign of a possible mechanism of saturation, due to the nonlinear behaviour of the scalar condensate. Thus it is due to many-body interactions. Also, it does not require a relativistic treatment of the nucleons of the matter. In these ways the saturation mechanism differs from that in the Walecka model.

Note also that the four quark condensates beyond the gas approximation include configurations where the two $\bar{q} q$ operators act on two different nucleons. This corresponds to the three-body forces in nuclear matter which are much discussed nowadays.

\section{Self-consistent scenario}

Thus SR enable a determination of the dependence of the effective mass $m *(\kappa(\rho))$. The pion propagator, renormalized by interactions with the matter depends on the nucleon effective mass $m^{*}$, and thus the condensate $\kappa$ depends on $m^{*}(\rho)$. It is reasonable to combine the SR with Finite Fermi System Theory at this point. Hence, a solution of the self-consistent equations provides the dependencies $m^{*}(\rho)$ and $\kappa(\rho)$. One should also include a calculation of the pion-nucleon coupling constant $g_{\pi N N}(\kappa(\rho))$, employing the SR. Note that the expectation value $\kappa(\rho)$ is an important parameter of the matter itself since it shows the degree of restoration of chiral symmetry. Inclusion of the hyperons will enable to trace the phase transitions of the matter. Solution of this self-consistent set of equation is a subject of future work.

\section{Concluding remarks}

Most of these results have been presented in the review [3]. Later [4] the approach was applied in an investigation of hyperons in nuclear matter. Note that the nucleon self energies in nuclear matter were also calculated in an alternative approach to the SR, based on the dispersion relations in the time component $q_{0}$ [5].

The authors thank Dr. Lucian Harland-Lang for reading the manuscript and acknowledge the support by the RFBR grants 11-02-00120-a and 12-02-00158-a.

\section{References}

[1] 1. M. A. Shifman, A. I. Vainshtein, and V. I. Zakharov, Nucl. Phys. B 147, 385 (1979); B. L. Ioffe, Nucl. Phys. B 188, 317 (1981); B. L. Ioffe, V. S. Fadin and L. N. Lipatov, Quantum Chromodynamics, Cambridge Univ. Press, Cambridge, 2010.

[2] E. G. Drukarev and E. M. Levin, Pis'ma ZhETF, 48, 307 (1988) (JETP Lett. 48, 338 (1988)).

[3] E. G. Drukarev, M. G. Ryskin, V. A. Sadovnikova, Phys. Atom. Nucl. 75, 334 (2012); ArXiv:1012.0394[nucl-th].

[4] E. L. Kryshen, Phys.Rev. C 84, 055205 (2011).

[5] T. D. Cohen, R. J. Furnstahl, D.K. Griegel, and X. Jin, Prog.Part. Nul. Phys. 35, 221 (1995). 